\title{
Der nacheiszeitliche Bergsturz im Kandertal (Schweiz): Alter und Auswirkungen auf die damalige Umwelt
}

\author{
Willy Tinner ${ }^{1}$, Petra Kaltenrieder ${ }^{1}$, Michael Soom ${ }^{2}$, Peter Zwahlen ${ }^{3}$, Martin Schmidhalter $^{4}$, \\ AdRIANO BOSCHETTI ${ }^{5} \&$ CHRISTIAN SCHLÜCHTER ${ }^{6}$
}

Key words: Landslides, rock falls, quaternary sediments, pollen analysis, macrofossil analysis, Corylus, Holocene, Alps

\section{ZUSAMMENFASSUNG}

Beim Bau des neuen AlpTransit Lötschberg Basistunnels wurden unter murgangartig verschwemmten Ablagerungen der alten Bergsturzmasse des Kandertals Stillwasserablagerungen mit zahlreichen organischen Resten und Torflagen gefunden. Die ${ }^{14} \mathrm{C}$-datierten Resultate der Pollen, Makrorest-, Holz- und Holzkohleanalysen ermöglichten eine Rekonstruktion der lokalen bis regionalen Umweltgeschichte. Ein Gewässer, vermutlich ein kleiner See, begann beim Tellenfeld in Frutigen um 8800 kal. Jahre v. Chr. zu verlanden. In der näheren Umgebung wuchs von 8800 v. Chr. bis 8000 v. Chr. ein Föhrenwald (Pinus silvestris), der reichlich mit Hasel (Corylus avellana) und anderen wärmeliebenden Gehölzen (Ulmen, Linden, Eichen; Ulmus, Tilia, Quercus) und Birken (Betula) durchsetzt war. Diese für die Nordalpen sehr frühe Bedeutung der Hasel ist durch ${ }^{14} \mathrm{C}$-datierte Corylus-Nussfragmente $\left(9310 \pm 50{ }^{14} \mathrm{C} \mathrm{BP}\right.$ 8722-8337 v. Chr.) belegt. Nach 8500 v. Chr. drängte die Hasel die Waldföhre allmählich zurück. Auf Grund der paläoökologischen Resultate muss angenommen werden, dass die Wälder um 7600 v. Chr. durch ein katastrophales Ereignis stark gestört wurden. Als Reaktion darauf kam es zu einer starken Zunahme der Waldbrände und es breiteten sich zuerst Farne und Gräser sowie wenig später Waldföhren aus. Das Gewässer wurde um 7100 v. Chr durch verschwemmtes Bergsturzmaterial zerstört. Der geomorphologische Befund deutet darauf hin, dass diese Ereignisse in engem Zusammenhang mit dem Hauptbergsturz im Kandertal stehen, der aussergewöhnliche Ausmasse hatte (ca. 800 Millionen $\mathrm{m}^{3}$ ). Die Zerstörung der lokalen Ökosysteme als Folge des Bergsturzes um 7600-7100 v. Chr. fiel in ein frühes holozänes Wärme- und Sonneneinstrahlungsmaximum, in dem es, wie vorgängige Untersuchungen in den Alpen und in anderen Gebirgen belegen, zu überdurchschnittlich vielen Hanginstabilitäten kam.

\section{ABSTRACT}

During the construction of the new AlpTransit railway line wetland sediments containing numerous fossils and peat layers were found below rockfall masses transported by debris flows. Radiocarbon-dated results of pollen, macrofossils, wood, and charcoal along with radiocarbon dating analysis were used to reconstruct the environmental history of the site. The wetland, originally probably a small lake, started to accumulate sediments at about $8800 \mathrm{cal}$. yr BC at Frutigen Tellenfeld. A pine forest (Pinus silvestris) admixed with hazel (Corylus avellana), other thermophilous arboreal taxa (Ulmus, Tilia, Quercus) and birch (Betula) grew in the surroundings of the lake. This very early importance of hazel is documented by ${ }^{14} \mathrm{C}$-dated Corylus nut fragments $\left(9310 \pm 50{ }^{14} \mathrm{C}\right.$ yr BP, 8722-8337 yr BC). After 8500 BC hazel expanded on the costs of pine. The palaeo records suggest that the forests were severely disturbed by a catastrophic event at around $7600 \mathrm{BC}$. In response, forest fires strongly increased and ferns and grasses expanded and then pine stands established. At ca. 7100 BC the lake was abruptly destroyed by rockfall masses transported by a debris flow. The geomorphic situation suggests that these events were closely related with the main Kander valley rockfall, which had an exceptional size (800 millions $\mathrm{m}^{3}$ ). Local environmental catastrophes as a consequence of the rockfall at 7600-7100 BC occurred during an early Holocene thermal and solar irradiation maximum. As documented by previous investigations, this period was characterised by pronounced slope instabilities in the Alps and elsewhere.

\section{Einleitung}

Die Ursachen von Hanginstabilitäten wie Bergstürze und Rutschungen werden in Anbetracht der globalen Klimaerwärmung zunehmend diskutiert, da kausale Zusammenhänge zwischen Klimaverlauf und Katastrophen bestehen (Dehn \&
Buma 1999). Das absolute Alter vorgeschichtlicher Bergstürze in den Alpen ist nur in wenigen Fällen bekannt (Lateltin et al. 1997, Dapples et al. 2003). Die meisten Bergstürze, die bislang mittels physikalischer Methoden (z.B. Radiokarbonmethode) datiert worden sind, weisen ein Alter von weniger als 12'000

\footnotetext{
1 Institut für Pflanzenwissenschaften, Abteilung Paläoökologie, Universität Bern, Altenbergrain 21, CH-3013 Bern, Switzerland.

2 Geotest AG, Birkenstrasse 15, CH-3052 Zollikofen, Switzerland.

3 Geotechnisches Institut AG, Seestrasse 7, CH-3700 Spiez, Switzerland.

4 Dendrolabor Wallis, Sennereigasse 1, CH-3900 Brig, Switzerland.

5 Archäologischer Dienst des Kantons Bern, Eigerstrasse 73, CH-3011 Bern, Switzerland.

${ }^{6}$ Institut für Geologie, Abteilung Quartärgeologie, Universität Bern, Baltzerstrasse 1-3, CH-3012 Bern, Switzerland.

E-mail Kontaktadresse: willy.tinner@ips.unibe.ch
} 


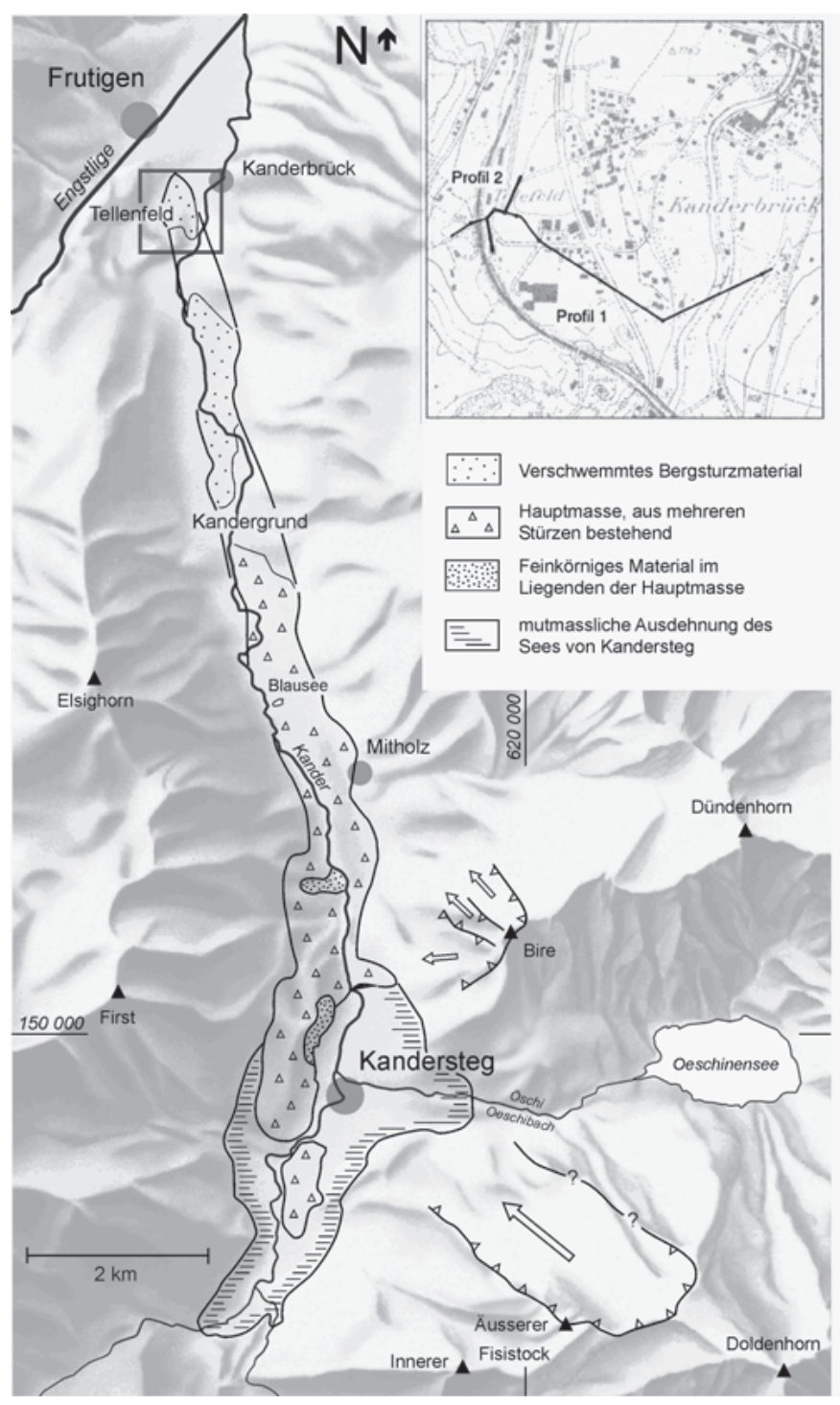

Fig. 1. Übersicht mit Verbreitung der Bergsturzablagerungen im Kandertal, ergänzt nach FURRER et al. (1993). Kartendaten Bundesamt für Landestopographie. Massstab 1:100000.

Reproduziert mit Bewilligung von Swisstopo (BAO56933)

Jahren auf (Lateltin et al. 1997, Dapples et al. 2003). Zur Zeit gibt es nur wenige chronologisch einwandfrei gesicherte Daten, welche belegen, dass es nach dem alpinen Eis-Kollaps der späten Würmeiszeit zu zahlreichen Bergstürzen kam (siehe Hantke 1978, von Poschinger \& Haas 1997, Becker et al. 2000, Dapples et al. 2003). Bei vielen grossen Bergstürzen (z.B. Kandertal, Flims) wird auf Grund geomorphologisch-geologischer Befunde ein späteiszeitliches Alter vermutet, ohne dass gesicherte Altersdatierungen vorliegen (z.B. Hantke 1978). Jüngste Untersuchungen am Flimser-Bergsturz, den umfangreichsten Sturzmassen innerhalb der Alpen, haben ein nachglaziales Ereignis aus dem Boreal nachgewiesen $\left(8000-8800 \mathrm{BP}{ }^{14} \mathrm{C}\right.$, Periode des ersten nacheiszeitlichen Klimaoptimums; von Po- schinger \& Haas 1997, Tinner \& Ammann 2001). Das Beispiel des Flimser-Bergsturzes belegt eindrücklich, wie heikel Altersinterpretationen nach rein geomorphologischen und selbst quartärstratigraphischen Argumenten sind (vgl. von Poschinger \& Haas 1997).

Bei Sondierbohrungen und dem Aushub für die Bauarbeiten zum Voreinschnitt des Portals Nord des Lötschberg Basistunnels im Tellenfeld bei Frutigen (Kanton Bern) wurden unter murgangartig umgelagerten Schottern ("Helke-Grien") der alten Bergsturzmasse des Kandertals Stillwasserablagerungen mit zahlreichen organischen Resten und Torflagen gefunden. In der vorliegenden Arbeit sollen paläobotanische und radiometrische Untersuchungen Auskunft über das Alter dieser Lockergesteinsablagerungen bei Frutigen-Tellenfeld liefern, welche Rückschlüsse auf das Mindestalter der alten, nacheiszeitlichen Bergsturzmasse im Kandertal ermöglichen. Zudem wird ein Bergsturzszenario und insbesondere die Wirkungen des Bergsturzes auf die Umwelt diskutiert. Mögliche Zusammenhänge zwischen Klimaerwärmung und Bergstürzen während des Holozäns werden kurz besprochen.

\section{Geologischer Überblick}

Der Talboden des Kandertals südlich von Frutigen ist von ausgedehnten Bergsturzablagerungen bedeckt. Die Hauptmasse des Bergsturzmaterials stammt vom Fisistock und von der Bire an der rechten Seite des Kandertals zwischen Mitholz und Kandersteg (Fig. 1). Die Sturzmassen lösten sich aus den Nordhelvetischen Sedimentdecken. Der Hauptsturz stammt aus der Doldenhorn-Decke (Fisistock) und ein Nebensturz kommt aus der Gellihorn-Decke (Bire). Die Felsmassen bestehen vorwiegend aus Formationen der Unterkreide, welche als monoton massige Kalke und Kieselkalke ausgebildet sind. Die Sturzmassen sind auf den schieferigen Öhrli-Mergeln der basalen Kreide abgerissen, die in beiden Fällen einen offen verfalteten dip-slope von durchschnittlich $27^{\circ}$ (Fisistock) bzw. $32^{\circ}$ (Bire) Gefälle bilden. Nach Beck $(1929,1952)$ lässt sich der Bergsturz in mehrere Phasen gliedern: 1) Ein älterer, spät- bis postglazialer Felssturz aus feinkörnigem Schutt, der zu einer temporären Abriegelung des Beckens von Kandersteg und zur Bildung eines Sees geführt hat. 2) Dieser See hat sich rasch entleert und zu einer murgangartigen Verfrachtung von Bergsturzmaterial bis in den Raum von Frutigen geführt (Fig. 1, 2). Beck (1929) beschreibt die Ablagerungen des alten Sturzes vom Bühlbad bei Kandersteg als "zu Brechsand und Staub zerriebenes Material". Solche Schuttmassen sind bei "Uf der Höh" (1300 m ü.M.) und am "Büel” (1200 m ü.M.) nördlich von Kandersteg aufgeschlossen. Die feinkörnigen Ablagerungen erstrecken sich talwärts von der Lokalität Schlossweide bis nach Achern und reichen ca. $3.5 \mathrm{~km}$ weiter als die grobblockigen, jüngeren Ablagerungen. Beck geht von der Annahme aus, dass der Hauptbergsturz (Fisistock) das Kandertal vorübergehend abriegelte. Darauf stauten sich Kander und Oeschine, wodurch das Wasser durch den porösen Schutt sickerte und einen Murgang auslöste, der bis gegen Frutigen vorstiess. 


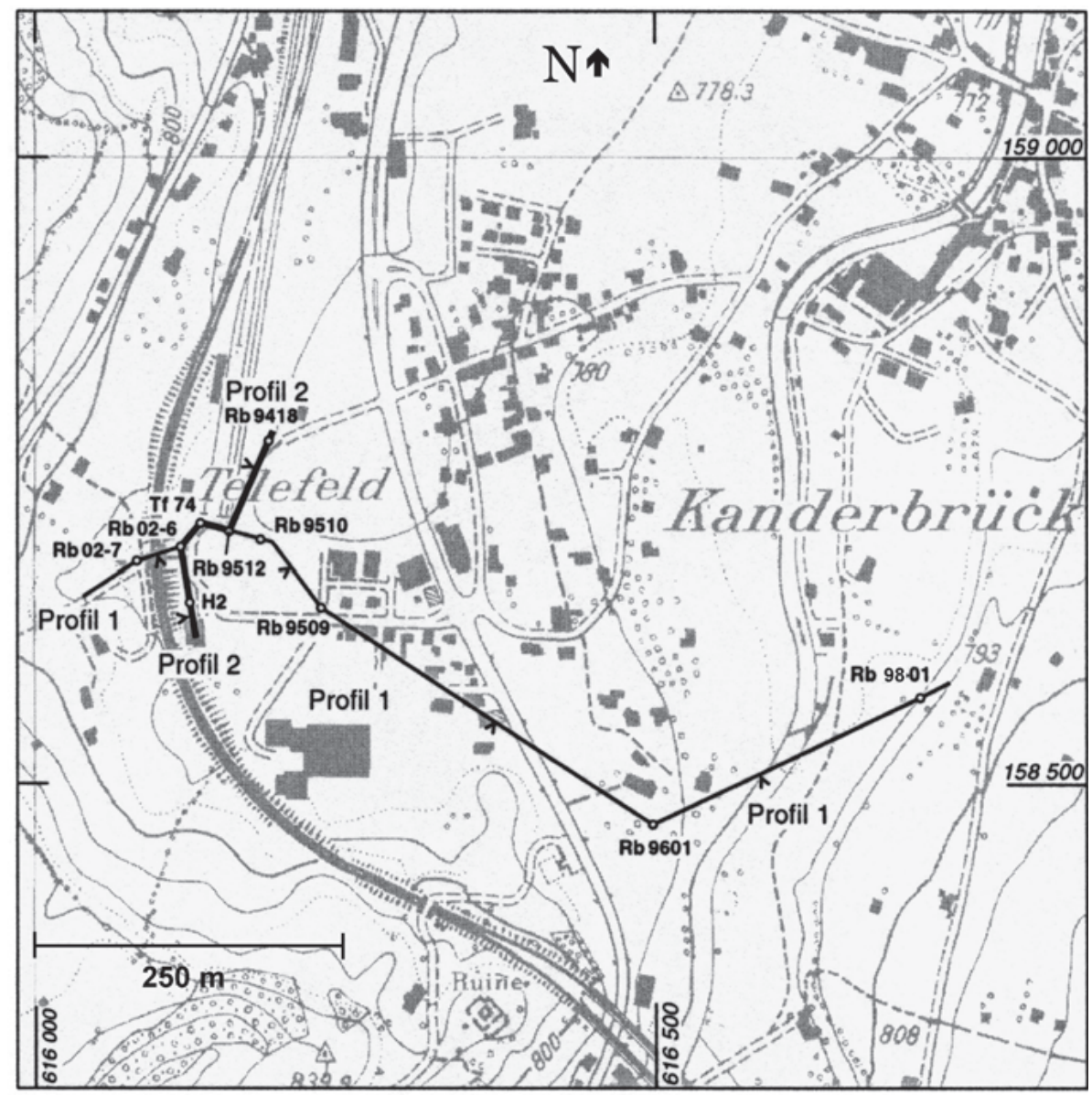

Fig. 2. Situation des quartären Schwemmbeckens Frutigen-Helke (Tellenfeld) mit den Bohrstandorten und den Profilspuren von Fig. 3. Distanz Koordinaten $158500 \mathrm{zu} 159000=500 \mathrm{~m}$. Kartendaten: UP10, () 1996 Vermessungsamt des Kantons Bern.
Beck $(1929,1952)$ schätzt für die alte Bergsturzmasse ein Volumen von ca. 800 Millionen $\mathrm{m}^{3}$ und eine Sturzbahn mit einer Höhendifferenz von $2000 \mathrm{~m}$ inkl. Murgang. Der Bergsturz von Kandersteg ist im Vergleich zu vielen dokumentierten Bergstürzen in den Alpen (ca. 285 nach Abele 1974) von überragender Bedeutung. Er ist zum Beispiel der siebentgrösste in Bezug auf das Volumen, hat die sechstlängste Fahrbahn (ca. $13 \mathrm{~km}$ ) sowie den grössten Fahrbahn-Höhenunterschied (Abele 1974).

\section{Untersuchungsgebiet}

Die nachfolgend beschriebenen Stillwasserablagerungen wurden im quartären Schwemmbecken in Altwassern der Kander (vermutlich in verlassenen Mäanderschlingen) abgesetzt. Das Becken liegt im Stauschatten des Engstlige-Schuttfächers (Flyschgeschiebe aus Adelboden), welcher die Kander gegen die rechte Talflanke abgedrängt und rückgestaut hat. In dieses Becken wälzte sich auch die Zunge der Bergsturz-Murgangablagerungen. Das Schwemmbecken im Tellenfeld bildet eine Schwemmlandebene, die an der linken Talseite etwa einen Kilometer südöstlich des Dorfkerns von Frutigen auf ca. $780 \mathrm{~m}$ ü.M. liegt. Das Gebiet befindet sich am Fuss der Hügelkuppen Tellenburg-Brunni-Büelen, die an alten Erosionsböschungen steil gegen das Schwemmland abfallen. Das Tellenfeld ist erst seit wenigen Jahrhunderten vor Überschwemmungen geschützt, nachdem die Kander verwuhrt und die Engstlige begradigt wurde.

Die mittlere Luftjahrestemperatur beträgt heute ca. $7^{\circ} \mathrm{C}$, die mittlere Jahresniederschlagsmenge ca. $1100 \mathrm{~mm}$. Das Klima kann als warm-gemässigt (Cfb nach Köppen, 1931) bezeichnet werden, mit mittleren Januartemperaturen um $-2^{\circ} \mathrm{C}$ und mittleren Julitemperaturen um $17^{\circ}$ C. In den montanen Talböden der Region finden sich Buchenwälder (Fagus silvati$c a)$, die an den Hängen oberhalb von $900 \mathrm{~m}$ ü.M. in TannenBuchenwälder (Abies alba, Fagus silvatica) und Tannenwälder übergehen (Welten 1982, Steiger 1995). In den Buchenwäldern sind Wärme liebende Bäume beigemischt (Quercus petraea, Ulmus scabra, Tilia cordata). Die Vegetation der Talböden wurde durch menschliche Tätigkeiten stark verändert. An vielen Talhängen der montanen Stufe (600-1400 m ü.M.) ist heute die Fichte (Picea abies) vorherrschend, vermutlich auch durch Forstpflanzungen bedingt.

Der auf Grund zahlreicher Sondierbohrungen für AlpTransit erkundete Schichtaufbau kann wie folgt zusammengefasst werden: Die murgangartig verfrachteten Bergsturzablagerungen überdecken am Hangfuss Moränenschichten des eis- 


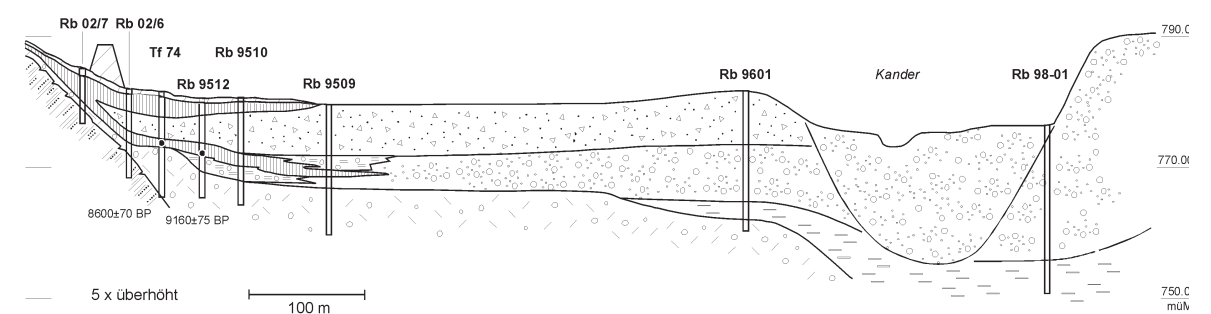

ssw

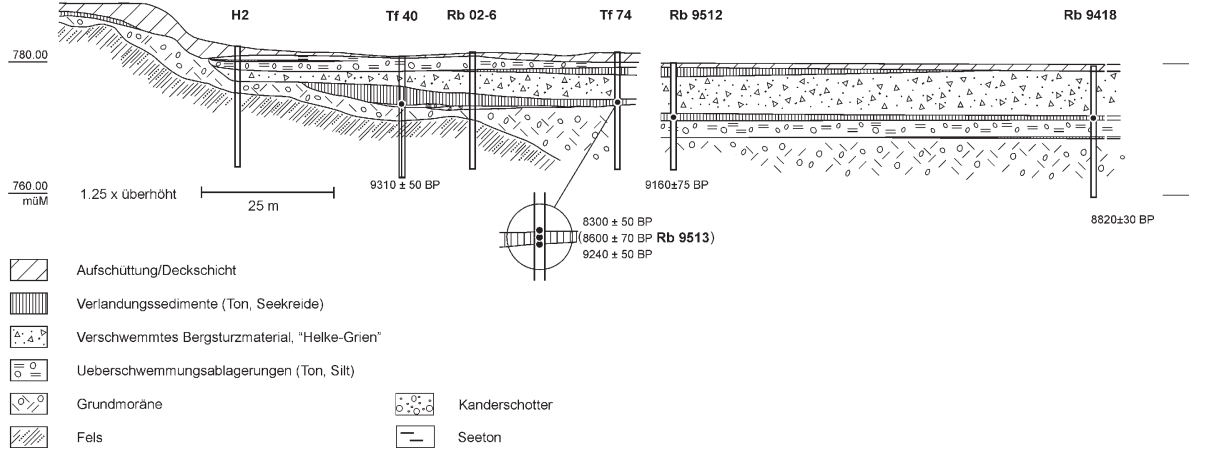

Fig. 3. Geologische Profile 1 und 2 im quartären Schwemmbecken Tellenfeld-Helke (Frutigen, Schweiz). Die ausgefüllten Kreise geben die stratigraphische Lage der Proben für die Radiokarbondatierungen an. Profilaufnahmen: M. Soom und P. Zwahlen. zeitlichen Kandergletschers, die auf dem Felsuntergrund aus Flyschgesteinen Nord- bis Ultrahelvetischer Herkunft liegen. Die Fels- und Moränenoberfläche fällt zur Talmitte hin flach $\mathrm{ab}$ und wird von altem Seeton überlagert. Über dem Seeton liegen Schotter der Kander, die gegen den Talrand hin mit feinkörnigen Stillwasserablagerungen und Verlandungssedimenten verzahnt sind. In einem eng begrenzten Gebiet unterhalb der Lokalität Brunni werden die Bergsturzablagerungen zudem von jüngeren Verlandungssedimenten überdeckt.

\section{Methoden}

\subsection{Bohrungen und Sedimente}

In den Jahren 1993/94 und 1995 wurden in Frutigen-Tellenfeld mehrere Sondierbohrungen durchgeführt. Die geographische Lage der für diese Untersuchung relevanten Bohrungen (Rb9418, Rb9510, Rb9512, Rb9513) ist in den Figuren 2 und 3 dargestellt. Die Sedimente wurden im Feld geologisch-geotechnisch aufgenommen, und es wurden einige ergänzende Korngrössenbestimmungen durchgeführt.

Die Proben von Sedimenten mit erhöhtem organischen Gehalt wurden den Bohrkisten im Juni 1995 entnommen (pers. Mitteilung D. Gutscher). Bis zu ihrer Untersuchung 2001 wurden sie in Kunstoffbehältern und Aluminiumfolie aufbewahrt. Im Jahr 2003 ergab sich die Gelegenheit, zwei
Aufschlüsse in der Alptransit-Baugrube (Tf40 und Tf74; Fig. 2 und 3) für ergänzende Analysen zu beproben.

\subsection{Radiokarbondatierungen}

Zur Radiokarbondatierung wurden nur pflanzliche Makroreste terrestrischen Ursprungs verwendet (s. Tab. 1). Eine Probe aus dem Kern Rb9418 wurde an der Universität Bern aufbereitet und datiert. Für zwei Proben aus den Kernen Rb9512 und Rb9513 erfolgte die für die Altersbestimmung erforderliche Aufbereitung des Probenmaterials im Radiokarbonlabor des Geographischen Institutes der Universität Zürich (GIUZ). Vier Proben aus den Aufschlüssen Tf40 und Tf74 wurden am Poznan Radiocarbon Laboratory in Polen aufbereitet. Die anschliessende Datierung wurde in Zürich (Institutes für Teilchenphysik, ETH) und in Poznan mittels AMS ("Accelerator Mass Spectrometry") durchgeführt. Die Radiokarbonalter wurden mit dem Programm calib4.3 (Stuiver et al. 1998) kalibriert.

\subsection{Pollenanalyse}

Für die Pollenanalyse wurde pro Probe $1 \mathrm{~cm}^{3}$ feuchtes Sediment unter Zugabe von Lycopodium Tabletten (Stockmarr 1971) aufbereitet. Die Zugabe von Lycopodium-Tabletten erlaubt die Berechnung von Pollenkonzentrationen (Pollenkör- 
Tabelle 1: Radiokarbondatierungen

\begin{tabular}{|c|c|c|c|c|c|}
\hline $\begin{array}{l}\text { Bohrung/ } \\
\text { Aufschluss }\end{array}$ & Tiefe $(\mathrm{cm})$ & Labornummer & Material & $\begin{array}{l}\text { Alter } \\
{ }^{14} \mathrm{C}(\mathrm{BP})\end{array}$ & $\begin{array}{l}\text { 95\% Konf. Interv. } \\
\text { kalibr. Alter }(\mathrm{BC}=\mathrm{v} . \text { Chr.) }\end{array}$ \\
\hline Rb9418 & $800-810$ & B-6621 & Holz & $8820 \pm 30$ & 8199-7749 BC \\
\hline Rb9512-Seekreide & $750-830$ & Uz-4593/ETH-24316 & Laubholz-Periderm & $9160 \pm 75$ & $8597-8241 \mathrm{BC}$ \\
\hline Rb9513-Seekreide & $710-750$ & Uz-4594/ETH-24317 & Laubholz-Periderm & $8600 \pm 70$ & $7763-7536 \mathrm{BC}$ \\
\hline $\mathrm{Tf} 40$ & $834-844$ & Poz-2514 & Corylus Nussfragmente & $9310 \pm 50$ & $8722-8337 \mathrm{BC}$ \\
\hline $\mathrm{Tf} 74$ & $265-255$ & Poz-6485 & Gehölz-Wurzel & $5390 \pm 40$ & $4337-4049 \mathrm{BC}$ \\
\hline $\mathrm{Tf74}$ & $689-694$ & Poz-2512 & Betula-Periderm & $8300 \pm 50$ & 7521-7143 BC \\
\hline Tf74 & $791-796$ & Poz-2496 & Pinus-Periderm & $9240 \pm 50$ & $8626-8290 \mathrm{BC}$ \\
\hline
\end{tabular}

Tabelle 2: Vereinfachte Sedimentbeschreibung der Rotationsbohrungen

\begin{tabular}{|c|c|c|c|c|c|}
\hline Tiefe (m) & $\begin{array}{l}\text { Rb9510 } \\
\text { Sediment }\end{array}$ & Tiefe $(\mathrm{m})$ & $\begin{array}{l}\text { Rb9512 } \\
\text { Sediment }\end{array}$ & $\begin{array}{l}\text { Rb9513 } \\
\text { Tiefe }\end{array}$ & Sediment \\
\hline $8.20-1.60$ & $\begin{array}{l}\text { Verschwemmtes Bergsturz- } \\
\text { material (Helke-Grien) }\end{array}$ & $7.30-2.00$ & $\begin{array}{l}\text { Verschwemmtes Bergsturz- } \\
\text { material (Helke-Grien) }\end{array}$ & $7.50-3.35$ & $\begin{array}{l}\text { Verschwemmtes Bergsturz- } \\
\text { material (Helke-Grien) }\end{array}$ \\
\hline $8.45-8.20$ & Seekreide, Torf $\bullet$ & $7.80-7.30$ & Seekreide, Kies, Kalkgyttja • & $7.50-7.10$ & $\begin{array}{l}\text { Siltige Seekreide, Torf } \\
\text { (schwärzlich) } \bullet\end{array}$ \\
\hline $10.00-8.45$ & Silt, Ton, Seekreide & $8.50-7.80$ & Siltiger Torf $\bullet$ & & \\
\hline $12.50-10.00$ & Kiesiger Silt, Pflanzenreste & $11.00-8.50$ & $\begin{array}{l}\text { Sandig-kiesiger Silt, } \\
\text { Pflanzenreste }\end{array}$ & $8.00-7.50$ & $\begin{array}{l}\text { Siltiger Sand-Sand, Torf, } \\
\text { Pflanzenreste }\end{array}$ \\
\hline $16.30-12.50$ & Grundmoräne & $16.00-11.00$ & Grundmoräne & $16.00-8.00$ & Grundmoräne \\
\hline
\end{tabular}

• = Probenahme für Radiokarbondatierungen, Pollen, Makroreste.

ner $\mathrm{cm}^{-3}$ ) und Polleninflux (Pollenkörner $\mathrm{cm}^{-2} \mathrm{yr}^{-1}$ ). Die Pollenaufbereitung folgte einem Standardverfahren (s. Moore et al. 1991) und umfasste eine Behandlung mit $10 \% \mathrm{HCl}, 10 \%$ $\mathrm{KOH}, 40 \% \mathrm{HF}$, Acetolyse, Präparation in Glycerin und Färben mit Fuchsin. Die Pollenkörner wurden im Durchlichtmikroskop bei 400-facher Vergrösserung mit Hilfe der Vergleichssammlung am Institut für Pflanzenwissenschaften der Universität Bern, von Identifikationsschlüsseln (z.B. Moore et al. 1991) und von Fotobänden (z.B. Reille 1992) bestimmt. Die Pollen-Nomenklatur folgt Moore et al. (1991). Für die Sedimente aus den Rotationsbohrungen wurde die Pollenanalyse nach Sedimenttyp getrennt durchgeführt (s. Resultate Pollenund Makrorestanalyse). In den Diagrammen sind die leeren Kurven 10 x überhöht.

\subsection{Makrorestanalyse}

Für die pflanzliche Makrorestanalyse wurden bei den Rotationsbohrungen Sedimentproben mit unterschiedlichem Volumen untersucht. Die Proben Rb9510-SK, Rb9512-SK, Rb9512KG und Rb9513-SK/KG umfassten $200 \mathrm{~cm}^{3}$, die Probe Rb9513-Torf $170 \mathrm{~cm}^{3}$, die Probe Rb9510-Torf $68 \mathrm{~cm}^{3}$ und die Probe Rb9512-Torf $32 \mathrm{~cm}^{3}$. Um einen Vergleich der Konzentrationen zu ermöglichen, wurden die Resultate nach der Analyse auf ein fiktives Volumen von $100 \mathrm{~cm}^{3}$ umgerechnet (Anzahl Makroreste $100 \mathrm{~cm}^{-3}$ oder Flächen in $\mathrm{mm}^{2} 100 \mathrm{~cm}^{-3}$ ). Für die Rotationsbohrungen wurde die Makrorestanalyse nach Se- dimenttyp getrennt durchgeführt (s. Resultate Pollen- und Makrorestanalyse). Bei den Aufschlüssen Tf40 und Tf74 umfassten die Proben $100 \mathrm{~cm}^{3}$ Volumen Sediment. Die Makroreste wurden mit Hilfe der Vergleichssammlung am Institut für Pflanzenwissenschaften der Universität Bern und mit Identifikationsschlüsseln bestimmt (z.B. Schoch et al. 1988). Die holzanatomischen Bestimmungen wurden mit Hilfe des Schlüssels für mikroskopische Holzanatomie (Schweingruber, 1990) durchgeführt. In den Diagrammen sind die leeren Balken $10 \mathrm{x}$ überhöht.

\subsection{Dendrochronologische Untersuchungen}

Aus den Sedimenten der Seeablagerungen fielen während den Bauarbeiten verschiedene grosse Gehölze (Megafossilien) von bis zu mehr als $50 \mathrm{~cm}$ Länge und mehr als $20 \mathrm{~cm}$ Durchmesser. Einige davon konnten nicht mehr genau den Sedimentschichten zugewiesen werden. Acht Proben wurden im Dendrolabor Wallis in Brig holzanatomisch untersucht (s. Schweingruber, 1990), bei vier erfolgte eine Ausmessung der Jahrringe.

\section{Resultate}

\subsection{Sedimente}

Die vier Bohrprofile und die zwei Baugrubenaufschlüsse weisen eine vergleichbare Lithostratigraphie auf (vgl. geologische 


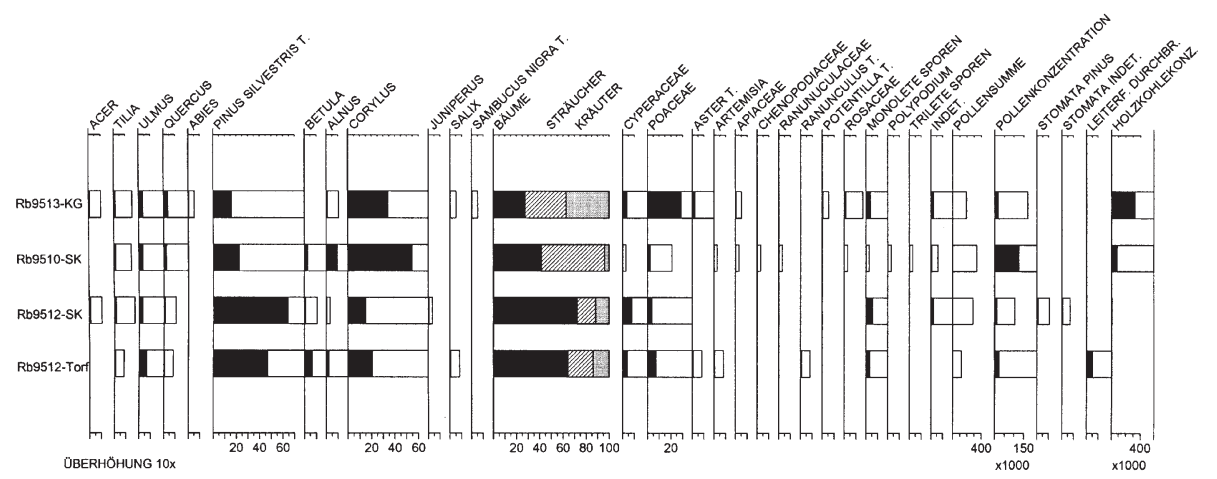

Fig. 4. Pollendiagramm der Rotationsbohrungen bei Frutigen-Tellenfeld. Pollen-, Sporen- und andere Mikrofossilienwerte in Prozenten, ausser Pollenkonzentration (Pollenkörner $\mathrm{cm}^{-3}$ ) und Holzkohlekonzentration (Teilchen $\mathrm{cm}^{-3}$ ). $\mathrm{KG}=$ Kalkgyttja, SK = Seekreide. Indet. = unbestimmt. Analyse: W. Tinner.
Profile in Fig. 3 und Falttafel). Auf Moränenmaterial (meist dichte Grundmoräne, untergeordnet blockige Obermoräne) folgen Überschwemmungsablagerungen und/oder Verlandungssedimente, auf denen verschwemmtes Bergsturzmaterial liegt. Darauf abgelagert sind erneut Verlandungssedimente und/oder eine Aufschüttungsschicht (s. Fig. 3 und Falttafel). Die Schichten mit Verlandungssedimenten umfassen unterschiedliche Sedimenttypen: Torf, toniger Silt, Kalkgyttja und Seekreide (Tab. 2).

Die zahlreichen Aufschlüsse, die im Verlauf der Bauarbeiten für Alptransit aufgenommen werden konnten, ergeben zusammen mit den Bohrbefunden folgendes Bild: Die Grundmoräne im Liegenden besteht aus einer feinkörnig, sandig-siltigen Grundmasse (Matrix) mit eingelagerten, z.T. glazial gekritzten Geschieben (zahlreiche Kalke, Kalksandsteine, z.T. Taveyannaz-Sandsteine, selten Gasterngranit). Die Grundmoräne ist sehr dicht gelagert (glazial vorbelastet) und matrixgestützt; ihre Oberfläche wirkt auf das Talgrundwasser als Stauhorizont. Am Hangfuss unterhalb der BLS-Linie und im Portalbereich des Basistunnels lagert über der Grundmoräne eine stellenweise braun verwitterte, kiesig bis grobblockige Obermoräne. In einem eng begrenzten, den steilen Talböschungen (ehemalige Prallufer der Kander) anschmiegenden Gebiet folgen über der Moräne Stillwassersedimente aus feingeschichtetem Silt mit mehreren Torflagen, eingeschwemmten Hölzern und Seekreide. Die Stillwasserablagerungen sind gegen die Talmitte zu mit (postglazialen) Kanderschottern verzahnt, wodurch ihre Mächtigkeit zur Lötschbergstrasse hin (Richtung Talmitte) kontinuierlich abnimmt. Über den Stillwassersedimenten folgt mit scharfer, erodierender Untergrenze verschwemmtes Bergsturzmaterial ("Helke-Grien").

Die Bergsturzablagerungen lassen sich in zwei Serien unterteilen: (1) Eine obere Serie (sog. "Helke-Grien", früher zur Strassenkofferung lokal in kleinen Mengen im Tellenfeld abgebaut) aus gut sortiertem Grobsand und Feinkies, durchwegs als kantige Komponenten ausgebildet, zu über $95 \%$ aus Kalksteinen bestehend, mit variablem Feinanteil und in freien Böschungen gut erkennbarer, fluviatiler Schrägschichtung. (2) Eine untere, chaotisch abgelagerte Serie, bestehend aus Feinbis Grobkies, vereinzelt auch mit Steinen und Blöcken, die in einer feinkörnigen Grundmasse aus Silt eingebettet sind. Diese sind gegen die Basis dicht gelagert und liegen erosiv auf der Basis aus Stillwassersedimenten und Kanderschotter. Der erosive Charakter der Basis der Bergsturzablagerungen wird durch Beobachtungen in der Ostwand der AlpTransit-Baustelle unterstützt, wo wenige Dezimeter oberhalb der Kontaktfläche Pakete von aufgearbeiteten Stillwasserablagerungen mit eingelagerten Holzstücken kartiert wurden.

Entlang der BLS-Linie wurden am Hangfuss Lockergesteine der oberen Serie festgestellt, die auf braun angewitterter Obermoräne lagern. Dies weist auf eine Bodenbildung vor der Ablagerung der Bergsturzmassen hin. Bei Brunni erscheint im Dach der Bergsturzablagerungen eine zweite Schicht aus Stillwasserablagerungen, die ebenfalls reich an organischem Material ist und sich bergseitig bis unter die BLS-Linie fortsetzt. Die Interpretation des bis $6 \mathrm{~m}$ mächtigen Kieskörpers (HelkeGrien) als murgangartig verschwemmtes Bergsturzmaterial ist u.a. aufgrund von Ausschlusskriterien zwingend. Die massige, im unteren Teil texturlose Ausbildung des Sedimentkörpers sowie die monotone Zusammensetzung der splitartigen Grobsand-Kies-Kornfraktionraktion aus schwarzgrauen Kalken und Kieselkalken schliessen Moränen- Fluss- und Hangschuttablagerungen aus. Die angedeutete Schrägschichtung im oberen feinerkörnigen Teil (Grobsand, wenig Feinkies) weist auf eine abschliessende Verschwemmung durch fliessendes Wasser hin, wobei auch dies nur über kurze Distanzen (kaum kantengerundeter Split). Damit ist zur Deutung des Kieskörpers nur ein turbulentes, kurzfristiges Ereignis denkbar, eben ein wassergesättigter Murgang, der seiner begrenzten inneren Dynamik entsprechend eine eng beschränkte Kornfraktion transportierte und im Tellenfeld absetzte. Bezüglich Materialzusammensetzung kommt als Einzugsgebiet nur die Bergsturzmasse von Kandersteg, mit seiner monotonen Zusammensetzung aus Kalken und insbesondere Kieselkalken der nordhelvetischen Unterkreide, in Frage. Der Kopf des Bergsturzes liegt $1 \mathrm{~km}$ südlich des Tellenfeldes und schliesst mit einer etwa $10 \mathrm{~m}$ hohen Rampe die Schwemmebene bei Frutigen-Rybrügg ab (vgl. Furrer et al. 1993).

\subsection{Radiokarbondatierungen}

Die Resultate der ${ }^{14} \mathrm{C}$-Analyse sind in Tabelle 1 zusammengefasst. Die datierten Proben aus den Sedimenten unterhalb des Helke-Griens fallen (mit 95\% Wahrscheinlichkeit) in den Zei- 
traum von 8722 bis 7143 v. Chr. Diese Resultate deuten darauf hin, dass die Moore, Tümpel oder Seelein, die zur Ablagerung der Verlandungssedimente (Torf, Seekreide, Kalkgyttja) unter dem verschwemmtem Bergsturzmaterial führten, während ungefähr 1600 Jahren bestanden (s. Diskussion). Die Datierung der zweiten Schicht aus Stillwasserablagerungen, die auf dem Helke-Grien abgelagert wurde, ergab ein Alter von 4337-4049 v.Chr. Da es sich beim datierten Material um eine GehölzWurzel handelt (es wurden keine anderen Makroreste terrestrischen Ursprungs gefunden), wird das Alter der oberen Stillwasserablagerung vermutlich unterschätzt (Durchdringung der Torfschichten durch Wurzeln jüngerer Bäume).

\subsection{Pollen}

Vier Proben aus den Rotationsbohrungen enthielten genügend Pollen zur quantitativen Analyse. Die Proben waren relativ pollenarm, trotzdem konnten durch intensive Analyse meistens Pollensummen von 200-400 erreicht werden. Die Proben Rb9512-SK und Rb9512-Torf weisen eine Dominanz von Pinus silvestris (Föhre) T. (T. = Pollentyp) auf (50-60\%). Der zweitwichtigste Pollentyp ist Corylus (Hasel). Nur niedrige Pollenprozentwerte erreichen Ulmus, Quercus, Tilia, Betula und Alnus (Ulme, Eiche, Linde, Birke und Erle). In der Probe Rb9512-SK wurde Pollen von Acer (Ahorn) gefunden. Die wichtigsten Vertreter der Nichtbaumpollen (NBP) und Sporen sind die Poaceae (Süssgräser) und Cyperaceae (Sauergräser). Höhere Werte erreichen ebenfalls die monoleten Sporen (Fig. 4). Der Fund von Pinus-Spaltöffungen (Stomata) in der Probe Rb9512-Seekreide fällt mit Maximalpollenwerten von Pinus silvestris T. zusammen und belegt die lokale Anwesenheit der Föhre. Die in der gleichen Probe gefundenen leiterförmigen Durchbrechungen stammen von Corylus-, Betula- oder Alnus-Holz.

In den Proben Rb9510-Seekreide und Rb9513-Kalkgyttja ist Corylus (Hasel) dominant und Pinus silvestris (Föhre) T. der zweitwichtigste Pollentyp. Ansonsten sind bei den Gehölzen, mit der Ausnahme von Pollenfunden von Abies (Weisstanne) in Rb9513-Kalkgyttja, kaum Unterschiede zu den Proben Rb9512-Seekreide und -Torf festzustellen. Bei den NBP fallen die sehr hohen Werte der Poaceae in der Probe Rb9513Kalkgyttja auf. Auch andere NBP (Aster T., Rosaceae) erreichen in dieser Probe relativ hohe Werte, so dass die Summe der Baumpollen (=Bäume und Sträucher) nur knapp über $60 \%$ steigt (in den anderen Proben $>80 \%$, s. Fig. 4).

Die Pollenresultate aus den Aufschlüssen Tf40 und Tf74 bestätigen die Resultate aus den Rotationsbohrungen und ermöglichen deren biostratigraphische Einordnung. Zwischen 860 und $840 \mathrm{~cm}$ dominiert bei Tf40 Pollen von Pinus silvestris (Föhre) T., der zweitwichtigste Pollentyp ist Corylus (Hasel) während Tilia, Ulmus, Quercus, Betula, Alnus glutinosa T. und Salix (Linde, Ulme, Eiche, Birke, Erle und Weide) nur niedrige Prozentwerte erreichen (Fig. 5). Die wichtigsten NBP sind Cyperaceae (Sauergräser) und Poaceae (Süssgräser). Besonders häufig sind in diesem Abschnitt Stomaten von Pinus. Diese Phase in Tf40 ist in guter Übereinstimmung mit den Tiefen 810

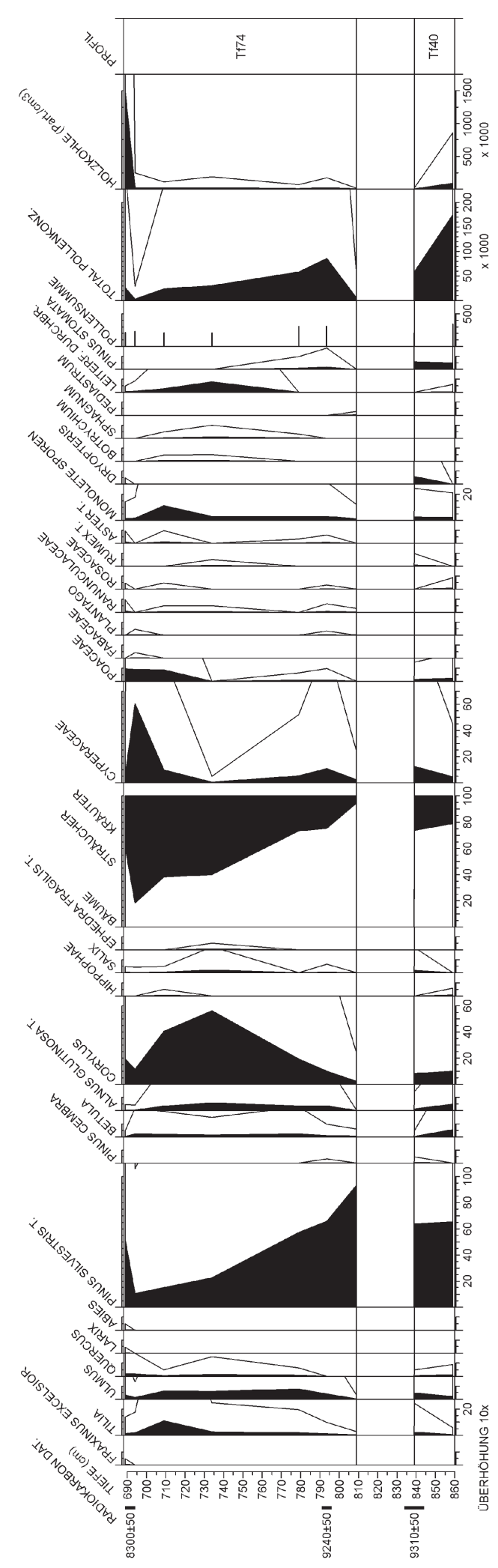

Fig. 5. Pollendiagramm der Aufschlüsse bei Frutigen-Tellenfeld. Pollen-, Sporen- und andere Mikrofossilienwerte in Prozenten, ausser Holzkohlekonzentration (Teilchen $\mathrm{cm}^{-3}$ ). Analyse: W. Tinner. 


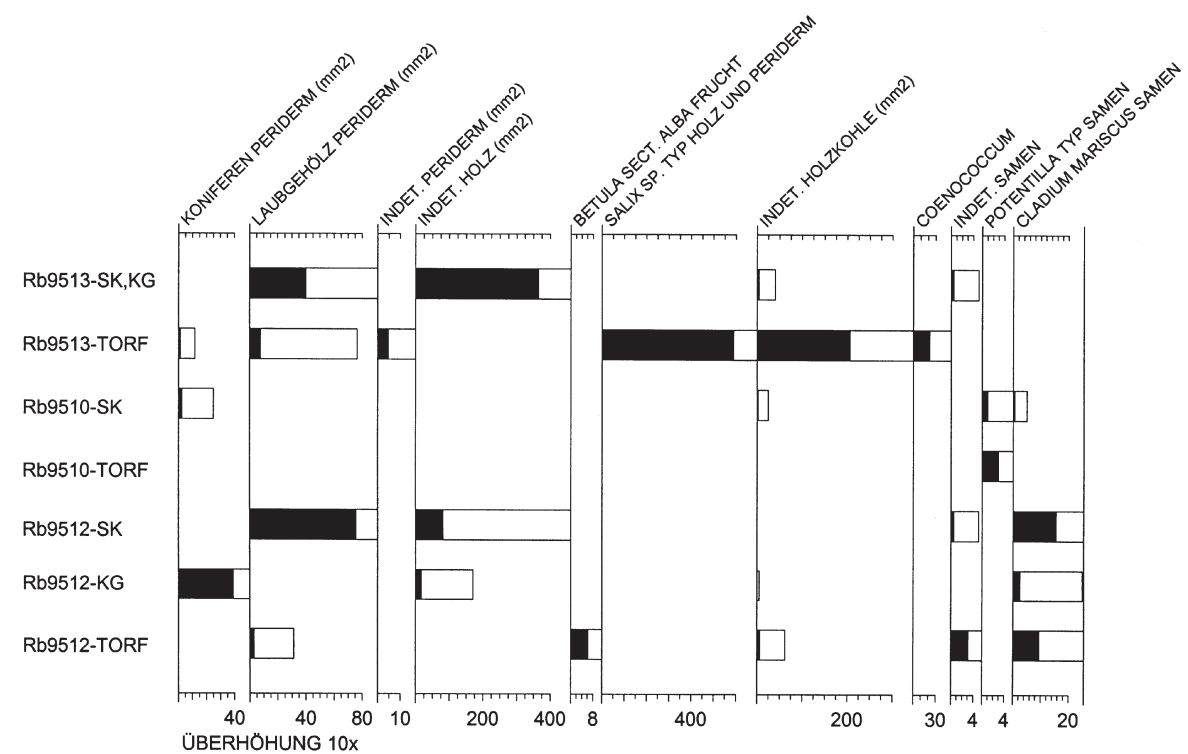

Fig. 6. Makrorestkonzentrationsdiagramm der Rotationsbohrungen bei Frutigen-Tellenfeld. KG = Kalkgyttja, SK = Seekreide. Früchte, Samen und Coenococcen (Anzahl 100 $\mathrm{cm}^{-3}$ ), Holz-, Holzkohle- und Peridermflächen $\left(\mathrm{mm}^{2} 100 \mathrm{~cm}^{-3}\right)$. Indet. $=$ unbestimmt. Analyse: P. Kaltenrieder. bis $760 \mathrm{~cm}$ aus dem Aufschluss Tf74 (Fig. 5) und den Rotationsbohrungen Rb95-12 Torf und Rb95-12SK (Fig. 4). Darüber (Tf74 760 bis $710 \mathrm{~cm}$ ) verdrängt Corylus Pinus silvestris T. als dominanten Pollentyp. Die Zunahme der leiterförmigen Durchbrechungen deutet auf eine vermehrte Ablagerung von Holz (vermutlich Corylus) während dieses Abschnittes. Im Gegensatz dazu, gehen die Pinus-Stomatenfunde allmählich zurück und verschwinden ab $734 \mathrm{~cm}$ Tiefe ganz. Als drittwichtigstes Gehölz erreicht Ulmus bei $780 \mathrm{~cm}$ maximale Pollenprozentwerte. Die Probe Rb9510-SK aus den Rotationsbohrungen (Fig. 4) kann eindeutig diesem Diagrammabschnitt zugeordnet werden. Im obersten Diagrammabschnitt von Tf74 (710-690 $\mathrm{cm}$ ) bricht Corylus ein und die Summe der Baumpollen nimmt von $>80 \%$ auf $<30 \%$ ab, hingegen erreichen die NBP Cyperaceae und Poaceae Maximalwerte. Die Zunahme dieser NBP ist mit kleineren Gipfeln bei anderen Kräutern (z.B. Fabaceae, Plantago, Aster T.) und bei den monoleten Sporen verbunden. In der obersten Probe drängt Pinus silvestris T. die NBP stark zurück, während Abies (Weisstanne) und Larix (Lärche) erstmals im Pollendiagramm erscheinen, und die mikroskopischen Holzkohlekonzentrationen hohe Werte erreichen (Fig. 5). Die Probe Rb9513-SK kann der Tiefe 709 cm im Aufschluss Tf74 zugewiesen werden. Obwohl die mikroskopischen Holzkohlekonzentrationen beim Tf74-Diagramm in dieser Tiefe im Gegensatz zu Rb9513-SK keine erhöhten Werte aufweisen, ist ein entsprechender Gipfel in den makroskopischen Holzkohlekonzentrationen (s. unten) deutlich erkennbar.

\subsection{Makroreste}

Alle sieben Proben aus den Rotationsbohrungen enthielten Makroreste pflanzlichen Ursprungs (Fig. 6). In jeder Tiefe (Rb9512, 10 und 13) wurden Koniferenperiderm (1.2 mm² 100 $\mathrm{cm}^{-3}$ - $\left.39 \mathrm{~mm}^{2} 100 \mathrm{~cm}^{-3}\right)$, Holzkohle $\left(0.5 \mathrm{~mm}^{2} 100 \mathrm{~cm}^{-3}->205\right.$ $\mathrm{mm}^{2} 100 \mathrm{~cm}^{-3}$ ) sowie einige nicht näher bestimmte Samen gefunden, wobei die Probe aus Kern Rb9510 allgemein die geringsten Werte aufweist. Die Probe Rb9512-Torf enthält als einzige Betula sect. alba (Baumbirken-) Früchte $\left(6100 \mathrm{~cm}^{-3}\right)$. Weiter finden sich wenig Laubgehölz Periderm und mehrere Samen der Wasserpflanze Cladium mariscus (Schneidebinse), welche hauptsächlich in dieser Tiefe vorkommen. Bei der Probe Rb9512-SK fällt die grosse Fläche an Laubgehölz-Periderm $\left(76 \mathrm{~mm}^{2} 100 \mathrm{~cm}^{-3}\right)$ auf. Potentilla (Fingerkraut) T. Samen kommen lediglich in den beiden Proben Torf und Seekreide des Kerns Rb9510 vor. Anhand ihrer Oberflächenstruktur kann es sich nicht um die einzige in diesem Gebiet nur im feuchten Lebensraum wachsende Potentilla palustris handeln. Somit stammen die Samen von einer terrestrischen Art dieser Gattung. Die weitaus höchsten Werte an Holz, Periderm und Holzkohle weisen die Proben von Rb9513 auf. Im Torf wurde ein ganzes Ästchen einer Weide (Salix sp.) abgelagert, was darauf hinweist, dass die Mutterpflanze ganz in der Nähe wuchs. Der in Rb9513-Torf gefundene Mykorrhizapilz der Gattung Coenococcum geht Symbiosen mit verschiedenen Pflanzengattungen ein, so dass keine genaueren Schlüsse über sein Vorkommen gezogen werden können. Nicht gezählt wurden Schneckenschalen, Moose, vegetative Cyperaceae-Reste (Samen analysiert) sowie nur dürftig erhaltene Makrorestfragmente, bei denen es sich eventuell um Holz handeln könnte.

Bei den Aufschlüssen Tf40 und Tf74 wurden ausser in 704-714 cm Tiefe in jeder Probe Reste (Zweige, Periderm, Holz, Nadeln, Kurztriebe, männliche Blüten, Flugsame) der Waldföhre, Pinus silvestris, gefunden (Fig. 7). Nur in der untersten Probe, in 854-864 cm Tiefe sind Chara Oogonien vorhanden. Armleuchteralgen sind kosmopolitische Wasserpflanzen und gedeihen in sehr unterschiedlichen Gewässern (Teichen, Seen und Fliessgewässer; Haas 1999). In den meisten Tiefen wurden Seggensamen (Carex sp.) gefunden, wobei die 
zwei untersten Proben (854-864 cm und 834-844 cm) die höchsten Konzentrationen aufweisen. Die Kurve ist für diesen Abschnitt in Übereinstimmung mit der Anzahl non-Sphagnum Laubmoose. Während die leichten Flugsamen der Baumbirke (Betula sect. alba) auch aus der weiteren Umgebung kommen können, zeigen die Funde von schwereren Fruchtschuppen und Zweigen derselben (854-864 cm; 834-844 cm; 689-694 cm), dass die Bäume ganz in der Nähe des Gewässers gewachsen sein müssen. Ebenfalls auf ein lokales Vorkommen von Corylus lassen die Funde von neun Haselnüssen (in 43 Stücken $100 \mathrm{~cm}^{-3}$ ) in $854-864 \mathrm{~cm}$ sowie von einer Haselnuss (zwei Stücke $100 \mathrm{~cm}^{-3}$ ) in 729-739 cm Tiefe schliessen. In den fünf Proben zwischen 814 und $704 \mathrm{~cm}$ war der Mykorrhizapilz der Gattung Coenococcum vorhanden. Im Seesediment wurde in 704 $\mathrm{cm}$ Tiefe ein ganzes Ästchen einer Weide (Salix sp.) mit einer Fläche von rund $2300 \mathrm{~mm}^{2} 100 \mathrm{~cm}^{-3}$ abgelagert. Die in fast allen Proben enthaltenen Samen, welche näher bestimmt werden konnten, gehören zu Kräuterpflanzen der Gattung Viola, einer terrestrischen Art von Potentilla und Eupatorium cannabinum. Letzterer, der Wasserdost, gedeiht an Ufern, in feuchten Wäldern und Riedwiesen. Im Sediment von 704-714 cm Tiefe wurden Reste einer Eichel (Quercus) festgestellt. Nur in den beiden obersten Proben, in einer Tiefe von 689-694 sowie 704-714 cm, sind Spuren von Feuer ersichtlich, in Form von Holzkohleteilchen, welche eine Gesamtfläche von 1626 bzw. $222 \mathrm{~mm}^{2} 100 \mathrm{~cm}^{-3}$ aufweisen.

\subsection{Dendrochronologie}

Die subfossilen Hölzer (s. Tabelle 4) sind auf Grund ihrer Fundlage ca. 10'000 Jahre alt, lassen sich aber vorerst nicht genauer datieren. Zwei der Bäume wurden mehr als 100 Jahre alt (Tabelle 4). Die Probe Nr. 90165, eine Ulme, wies 115 Jahre auf. Die äussersten Jahre waren der Engringigkeit (äusserst schmale Jahrringe) wegen, nicht mehr messbar. Es war kaum Spätholz vorhanden. Bei der Probe Nr. 90159 (Föhre) liessen sich die ersten 150 Jahre messen, sie wies aber vermutlich gegen 200 Jahre auf.

\section{Diskussion}

\subsection{Chronologie und Sedimente}

Die Radiokarbondatierungen und die Sedimentabfolge deuten darauf hin, dass sich in Frutigen-Tellenfeld vor 8800 v. Chr. in Altläufen der Kander einzelne Seelein (Hinterwässer) gebildet hatten (Überschwemmungssedimente oberhalb der Grundmoräne Rb9510, Rb9512, Rb9513, Tab. 2), die um 8800 v. Chr. allmählich zu verlanden begannen und spätestens um 7100 v. Chr. von verschwemmtem Bergsturzmaterial überschüttet wurden. Weil die Seeablagerungen eng mit fluviatilen Kanderablagerungen vergesellschaftet sind, neigen wir zur Annahme, dass es sich dabei um einen Stillwasserbereich der Kander im Staubecken oberhalb des Engstligen-Schuttfächers handelt.

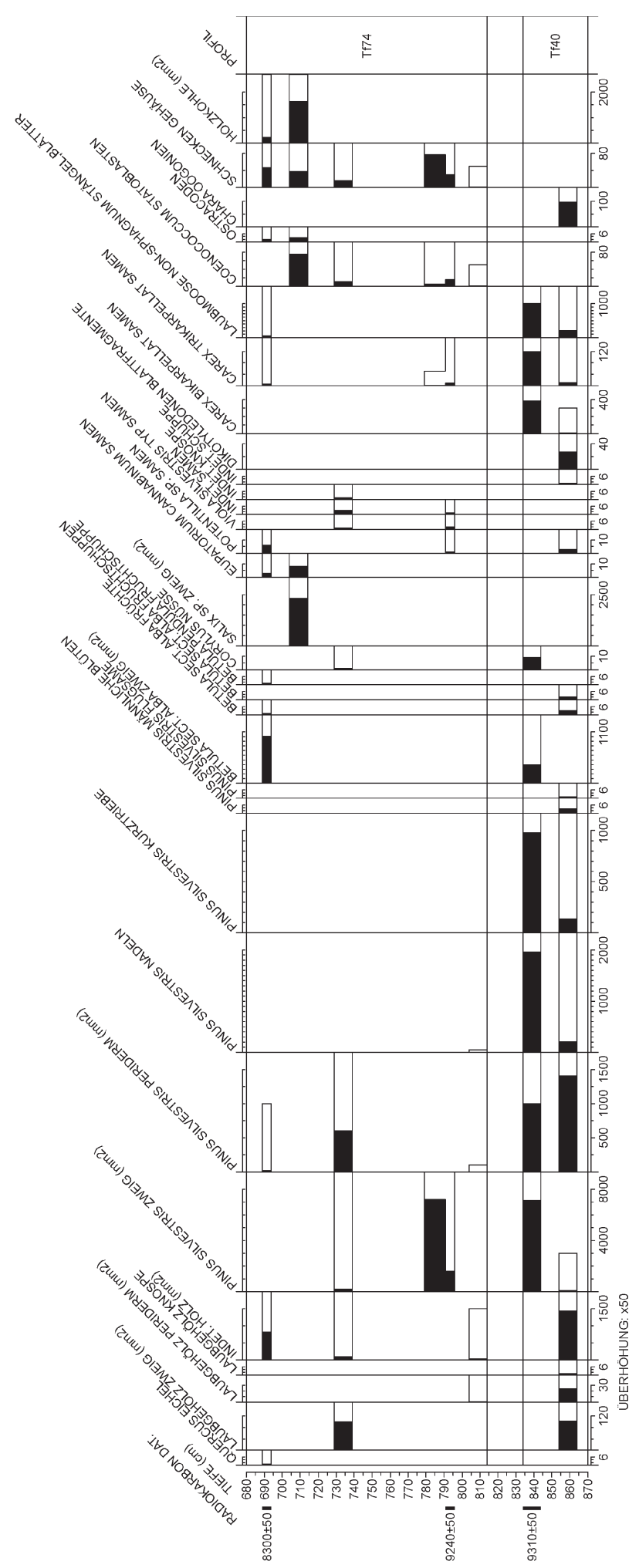

Fig. 7. Makrorestkonzentrationsdiagramm der Aufschlüsse bei Frutigen-Tellenfeld. Früchte, Samen, Eicheln, Nüsse, Nadeln, Kurztriebe, männliche Blüten, Fruchtschuppen, Knospen, Schuppen, Blattfragmente, Stängel, Blätter, Chara Oogonien, Schnecken und Coenococcen (Anzahl 100 $\mathrm{cm}^{-3}$ ), Holz- und Peridermflächen $\left(\mathrm{mm}^{2} 100 \mathrm{~cm}^{-3}\right)$. Indet. = unbestimmt. Analyse: P. Kaltenrieder. 
In Bezug auf die Lithostratigraphie weichen die Aufschlüsse und die Rotationsbohrungen v.a. durch das Auftreten bzw. Fehlen von Überschwemmungssedimenten zwischen der Grundmoräne und den Verlandungssedimenten voneinander ab. Da diese Überschwemmungssedimente reichlich Kies und Sand aufweisen, ist anzunehmen, dass deren Ablagerung schnell erfolgte, vermutlich innerhalb weniger Jahrzehnte. Stille Gewässer können sehr schnell von der Vegetation erobert werden und verlanden (Frey \& Lösch 1998). Dabei bilden sich je nach Wasserstand und Kalziumkarbonatanteil Gyttja, Seekreide oder Torf (so sind z.B. am Lago di Origlio in den Südalpen in den letzten 80 Jahren über $50 \mathrm{~cm}$ Feindetritus-Gyttja abgelagert worden; Tinner et al. 1998).

Die beiden Proben Rb9512-SK und Rb9512-Torf lassen sich aufgrund benachbarter und absolut datierter Biostratigraphien sowie der durchgeführten ${ }^{14} \mathrm{C}$-Datierungen in die Zeit um 8600 v. Chr. einordnen, wobei Rb9512-Torf auf Grund litho- und pollenstratigraphischer Merkmale (Torf, höhere Betula-Werte) wahrscheinlich etwas älter als Rb9512-SK ist. Wegen der hohen Corylus-Werte ist anzunehmen, dass Rb9512-Torf nicht älter als 9100 v. Chr. ist $\left(9650{ }^{14} \mathrm{C}\right.$ Jahre BP, s. van der Knaap \& Ammann 1997, Lotter 1999), ihr wahrscheinlichstes Alter ist 87008600 v. Chr. Die beiden Proben Rb9510-SK und Rb9513-KG lassen sich aus denselben Gründen dem Zeitabschnitt 8500-7400 v. Chr. zuordnen, wobei letztere auf Grund pollenstratigraphischer Merkmale (Corylus-, Pinus-, Abies - Werte) wahrscheinlich jünger als erstere ist. Diese Interpretation wird durch die ${ }^{14} \mathrm{C}$-datierten Diagramme der Aufschlüsse Tf40 und Tf74 unterstützt. Die zunehmenden Corylus-Werte an der Basis der Verlandungssedimente von Tf40 und Tf74 gerade oberhalb der Grundmoräne deuten zum Beispiel ebenfalls darauf hin, dass das Gewässer in den Jahrhunderten nach 9100 v. Chr. zu verlanden begann und auf Grund der ${ }^{14} \mathrm{C}$-Resultate von $\mathrm{Tf} 40$ und Tf75 (Tab. 3) erscheint ein Beginn der Verlandung um 8800 v. Chr. am wahrscheinlichsten.

\subsection{Vegetationsgeschichte}

Um das verlandende Gewässer wuchs zwischen ungefähr 8800 und 8000 v. Chr. ein Föhrenwald (Pinus silvestris), der reichlich mit Hasel (Corylus avellana) und anderen wärmeliebenden Gehölzen (Ulmus, Tilia, Quercus; Ulmen, Linden, Eichen) und Birken (Betula) durchsetzt war. Zu dieser Zeit herrschte überall im Schweizerischen Mittelland und in den Tallagen des Berner Oberlands dieser Vegetationstyp vor (z.B. Welten 1982, Ammann et al. 1996, Van der Knaap \& Ammann 1997, Lotter 1999). An den Ufern des Gewässers und in den moorigen Teilen des Tellenfelds wuchs ein Schneidebinsengürtel (Cladium mariscus). Im Wasser kamen Armleuchteralgen (Chara Oogonien, Fig. 7) und Grünalgen (Pediastrum) vor. Andere Wasserpflanzen sind weder durch die Pollenanalyse noch durch die Makrorestanalyse eindeutig belegt. Es ist aber wahrscheinlich, dass ein Teil des Cyperaceae- und PoaceaePollens von Sauer- und Süssgräsern stammte, die in der Verlandungszone wuchsen.
Gestützt auf regionale Pollenstratigraphien (s. Welten 1982, Ammann et al. 1996, Van der Knaap \& Ammann 1997, Lotter 1999) ist anzunehmen, dass sich spätestens ab $8500 \mathrm{v}$. Chr. die Hasel zunehmend auch im Mittelland und in den Voralpen ausbreitete, vermutlich im Unterholz sowie in kronenbildenden Beständen, während die Waldföhre merklich zurückgedrängt wurde (s. auch Polleninfluxwerte und Makrorestfunde am Soppensee, wobei die Hasel in den Makroresten fehlt; Lotter 1999). Die ${ }^{14}$ C-datierten Corylus-Nussfragmente (87228337 v. Chr., Tab. 1) sind somit ein sehr früher Beleg für die in späteren Jahrhunderten noch zunehmende Bedeutung der Hasel in den Nordalpen. Die Begünstigung der Hasel (Corylus) gegenüber den anderen wärmeliebenden Gehölzen, der Föhre (Pinus) und Birke (Betula) während des Frühholozäns (ca. 8600-6200 v. Chr.; Lotter 1999) ist vermutlich auf deren ausgeprägte Trockenheitsresistenz zurückzuführen (Tinner \& Lotter 2001). Im Zeitabschnitt von 8000 bis 7000 v. Chr. war die Sonneneinstrahlung gegenüber heute auf der Nordhalbkugel während des Sommers höher und während des Winters niedriger (Kutzbach \& Webb III 1993). Die Folge davon waren heiss-trockene Sommer, kalte Winter, eine ausgeprägte Niederschlagsarmut (Jahresniederschlag $<500-700 \mathrm{~mm}$ im Schweizer Mittel- und Oberland) und niedrige Seespiegel (Guiot et al. 1993). Die klimatische Situation war mit jener der heutigen alpinen Trockentäler (z.B. Wallis) vergleichbar, nur, dass die Winter kälter ausfielen (Tinner \& Lotter 2001).

Die vermutlich jüngste Pollenprobe Rb9513-Kalkgyttja hat einen stark erhöhten NBP-Anteil (37.4\%), der auf ausgeprägte Störungen der Waldvegetation hinweist (s. Zoller \& Haas 1995). Ähnlich weisen auch die obersten Proben in Tf74 stark erhöhte NBP-Werte auf. Es ist nicht ganz auszuschliessen, dass die hohen NBP-Werte in Tf74 $694 \mathrm{~cm}$ zum Teil auf Verlandungsprozesse zurückzuführen sind, da diese an vielen Standorten mit erhöhten Cyperaceae-Prozentwerten verbunden sind (Welten 1982). Allerdings zeigt das Makrorestdiagramm, dass dieser Cyperaceae-Pollen-Gipfel keine Entsprechung in den Makroresten hat, was gegen eine lokale Ausbreitung der Sauergräser spricht. Eine Zunahme der NBP rein auf Grund von Verlandungsprozessen scheint auch deshalb unwahrscheinlich, weil die Cyperaceae-Werte in den Pollenproben Rb9513-KG, Tf74 $689 \mathrm{~cm}$ und Tf74 $709 \mathrm{~cm}$ nicht wesentlich erhöht sind. Zudem sind Verlandungsprozesse, die zu einer lokalen Zunahme der Cypearaceae führen, an den meisten untersuchten Standorten mit Torf- und Moorbildung verbunden (s. z.B. Welten 1982, Tinner \& Theurillat 2003), die für diese Proben fehlt (Seekreide und Kalkgyttja).

Auf Grund der ungefähren Grösse des ehemaligen Beckens bei Frutigen-Tellenfeld und von Modellannahmen über Polleneinzugsgebiete, kann abgeleitet werden, dass das Störungssignal (starke Zunahme NBP) in den jüngsten Proben aus einer Entfernung von $400 \mathrm{~m}$ (30-45 \% des Pollens, Sugita 1994) bis $10 \mathrm{~km}$ (70-100 \% des Pollens, Bradshaw \& Webb 1985, Schwartz 1989) stammte. Da das Signal in vier Pollenproben aus zwei unterschiedlichen Profilen belegt ist, erscheint die folgende versuchsweise Interpretation angebracht: 
Tabelle 3: Vereinfachte Sedimentbeschreibung der Aufschlüsse

\begin{tabular}{llll}
\hline Tiefe $(\mathrm{m})$ & Sediment Tf40 & Tiefe $(\mathrm{m})$ & Sediment Tf74 \\
\hline $0-0.50$ & Humus und Oberboden & $0-1.20$ & Deckschicht/Aufschüttung \\
$0.50-3.00$ & Ton, Silt, Seekreide, Torf & $1.20-3.10$ & Ton, Silt, Seekreide, Torf \\
$3.00-6.60$ & Verschwemmtes Bergsturzmaterial (Helke-Grien) & $3.10-6.90$ & Verschwemmtes Bergsturzmaterial (Helke-Grien) \\
$6.60-8.65$ & Seekreide (mit Torf) & $6.90-8.15$ & Seekreide (mit Torf) \\
$8.65-9.20$ & Grundmoräne & $8.15-9.00$ & Grundmoräne \\
\hline
\end{tabular}

Tabelle 4: Holzartenbestimmung und Lebensdauer subfossiler Hölzer (Megafossilien)

\begin{tabular}{llll}
\hline Probe & Labornummer & Taxon & Lebensdauer (Jahre) \\
\hline Tfbg1 & 90156 & Ulmus sp. & 115 \\
Tfbg2 & & Pinus silvestris/mugo & 63 \\
Tf40a1 & 90158 & Pinus silvestris/mugo & 43 \\
Tf40a2 & 90157 & Pinus silvestris/mugo & \\
Tf40b & & Pinus silvestris/mugo & \\
Tf40c & & Salix sp. & $>150$ \\
Tf40 160-170 cm & 90159 & Pinus silvestris/mugo & Pinus silvestris/mugo \\
Tf74a & & Ping & \\
\hline
\end{tabular}

Herkunft der Hölzer. Tfbg1 und Tfbg2: Probenahme Soom, 11.09.2002 in grauer Siltschicht beim Tellenfeld, ca. 8 m Tiefe Tf40a: Probenahme Zwahlen 19.11.02 bei unterster Torflage, ca. $8.25 \mathrm{~m}$ Tiefe. Tf40b und Tf40c: Probenahme Zwahlen 19.11.02 zwischen unterster Torflage und Seekreide, ca. 8.10 m Tiefe. Tf40 160-170 cm: Probenahme Kaltenrieder, 5.3.2003. Tf74a: Probenahme Zwahlen: 24.2.2003 unterste Torflage, ca. 8.10 m Tiefe.

Wahrscheinlich wurden mehrere Hektaren Föhren-Haselstände im Umkreis von etwa einem Kilometer zerstört (mindestens 8-10 mal die Fläche des Tellenfeldgewässers, s. Sugita et al. 1997, d.h. mindestens fünf Hektaren, s. Fig. 1 und 2). Einige makroskopische (Fig. 6 und 7) und sehr viele mikroskopische (Fig. 4 und 5) Holzkohlefunde weisen darauf hin, dass die Störung mit Waldbränden verbunden war. Eine anthropogene Veränderung der Vegetation durch Feuer kann nicht ganz ausgeschlossen werden, eine natürliche Ursache ist aber am Naheliegendsten, da grossräumige Waldbrände mit einer derartigen Wirkung auf die Vegetation für das Schweizer Paläolithikum und Mesolithikum bisher unbekannt und erst für die Zeit nach 5500 v. Chr. dokumentiert sind (s. Tinner et al. 1999, Tinner et al. 2000, Tinner et al. unpubl.). Nach der Zerstörung der Wald- und Strauchbestände scheint sich vor der Vernichtung des Gewässers durch das verschwemmte Bergsturzmaterial kurzfristig ein Föhrenwald ausgebildet zu haben. Diese ausgeprägte Massenausbreitung der Waldföhre (Pinus silvestris) um 7100 v. Chr. passt wie die starke Öffnung der Wälder und die Zunahme der Waldbrände nicht in die sonst recht einheitliche regionale Vegetationsgeschichte der Alpen und des Schweizer Mittellands und deutet auf eine bedeutende und nachhaltige Störung der lokalen bis regionalen Vegetation hin.

\subsection{Bergsturzszenario}

Altläufe der mäandrierenden Kander im aufgestauten Schwemmbecken oberhalb des Engstligen-Schuttfächers führten vor 8800 v. Chr. zur Bildung eines Seeleins. Das allmählich verlandende Gewässer (Torfbildung) bestand während mindestens 1700 Jahren. Um 7600 v. Chr. ereignete sich der Bergsturz oberhalb des Tellenfelds und zerstörte einen beträchtlichen Teil der Vegetation. Diese Katastrophe ist wahrscheinlich in der Pollenprobe Rb9513-SK und im obersten Diagrammabschnitt des Aufschlusses Tf74 festgehalten. Die Waldzerstörung führte zur Bildung von überdurchschnittlich viel Totholz (evt. ersichtlich an den hohen Holz- und Peridermkonzentrationen in den Makrorestproben des Kerns Rb9513, Fig. 6), das in der Folge hypothetisch zu einer Zunahme der Waldbrände führte (sehr hohe mikroskopische Holzkohlekonzentration in Rb9513-SK und in der Makrorestprobe Rb9513-Torf, die dazugehörige Pollenprobe Rb9513-Torf besteht fast ausschliesslich aus Holzkohle und die Pollen- und Makrorestproben aus Tf74 weisen ebenfalls hohe Holzkohlewerte auf). Die Zunahme an Totholz könnte eine direkte Folge des Bergsturzes sein: Von zahlreichen alpinen Bergstürzen ist bekannt, dass sie zu massivem Windschlag am Wald geführt haben (z.B. Heim 1932). Es ist auch denkbar, dass durch intensiven Funkenschlag z.B. am Kieselkalk während des Sturzprozesses die angefahrenen Wälder entzündet wurden. Insbesondere im Kieselkalk sind Lagen bekannt, die durch Eisenerze wie Hämatit, Limonit und Pyrit imprägniert sind. Letzteres (Eisensulfid) kann leicht Funkenschlag erzeugen.

Nach der Waldzerstörung durch den Bergsturz, erfolgte zusammen mit der Zunahme der Waldbrände, eine Ausbreitung der Gräser und Farne (Poaceae und monolete Sporen in Fig. 5). Nur wenige Jahrzehnte später breiteten sich (vermutlich auf dem Bergsturzschutt) Waldföhren aus (dokumentiert 
durch den steilen Anstieg von Pinus silvestris in der obersten Probe von Tf74, Fig. 5). Tatsächlich ist heute nach einer frühen Sukzessionsphase, die v.a. durch Gräser und Farne dominiert wird, die Waldföhre der Charakterbaum vieler, auch älterer Bergsturzlandschaften, denn sie ist dem stark wasserdurchlässigen, flachgründigen und groben Bergsturzgelände besser angepasst als die meisten übrigen Waldbäume (Abele 1974). Nach dem Bergsturz staute sich im Kandertal bei Kandersteg hinter dem Bergsturzmaterial ein See, der nach kurzer Zeit wieder auslief. Das Bergsturzmaterial wurde von den Wassermassen zum Tellenfeld geführt und deckte das Seelein zu. Unser Szenario entspricht weitgehend dem Ablauf, der von Beck $(1929,1952)$ auf Grund des geomorphologischen Befunds vorgeschlagen wurde.

Diese Interpretation erlaubt die versuchsweise Datierung des Bergsturzes. Wird eine konstante Sedimentationsrate in Tf74 angenommen $(10.7 \mathrm{Jr} / \mathrm{cm})$, so ereignete sich der Bergsturz auf Grund der indirekten biostratigraphischen Indikatoren (Störung der Waldvegetation, Waldbrände) um $7600 \mathrm{v}$. Chr. (709 cm in Tf74, Fig. 5), während 200-500 Jahre später ein Murgang (vermutlich als Folge der nach dem Bergsturz erfolgten Seebildung bei Kandersteg) das Tellenfeld bei Frutigen zuschüttete $(690 \mathrm{~cm}$ in Tf74, s. auch Tab. 1, extrapoliertes Alter: 7400-7100 BC). Aus den neu gewonnenen paläoökologischen Daten sowie der allgemeinen geomorphologischen $\mathrm{Si}$ tuation (Fig. 1) vermuten wir, dass es sich beim ersten Ereignis um den Hauptbergsturz im Kandertal handelte. Eine abschliessende Betrachtung dieser offenen Frage würde eine direkte Datierung der Hauptbergsturzmasse an mehreren Standorten erfordern.

\section{Schlussfolgerungen}

Der Bergsturz im Kandertal ereignete sich zwischen 9000 und 7000 v.Chr, wahrscheinlich um 7600 v.Chr (Präboreal bis Boreal). Um 8000-7500 v.Chr wurde im Boreal ein frühes nacheiszeitliches Klimaoptimum in den Alpen erreicht (Haas et al. 1998, Tinner \& Ammann 2001, Tinner \& Theurillat 2003). Die Zeit zwischen 9500 v. Chr. und 6500 v. Chr. wies im Sommer auf der Nordhemisphäre eine um $8 \%$ erhöhte Sonneneinstrahlung gegenüber heute auf (Kutzbach \& Webb III 1993). Dies führte zu höheren Sommertemperaturen in den Alpen (s. z.B. Wick \& Tinner 1997, Tinner \& Theurillat 2003). Neuere Untersuchungen zeigen, dass dieser allgemeine Verlauf der Sonneneinstrahlungskurve durch kurzfristige Veränderungen kleinerer Schwankungsbreite überlagert war. Schwankungen der Kosmonuklide ${ }^{14} \mathrm{C}$ und ${ }^{10} \mathrm{Be}$ belegen, dass zwischen zwei mit dem Maunder-Minimum vergleichbaren Strahlungsminima um 8100 und 7400 v. Chr., die Sonneneinstrahlung zwischen 8000 und 7500 v. Chr. ein Maximum ereichte (Muscheler et al. 2000). Vermutlich bewirkte die zeitweilig erhöhte Sonneneinstrahlung die in den Alpen vielerorts festgestellte Wärmephase um 8000-7500 v. Chr.
Es gibt deutliche Hinweise darauf, dass in den Alpen und anderen Gebirgen Bergstürze mit holozänen Klimaänderungen (z.B. Lateltin et al. 1997, Alexandrowicz \& Alexandrowicz 1999, Trauth et al. 2000, Dapples et al. 2003) und anderen naturräumlichen Ursachen (z.B. Erdbeben, s. Tibaldi et al. 1995, Hermanns et al. 2000) zusammenhingen. Zwei klimatische Faktoren waren für die Auslösung von Bergstürzen und -rutschen in Hochgebirgen entscheidend: erhöhte Niederschlagsmengen (in Form von Schnee und Wasser) und überdurchschnittliche (Sommer)-Temperaturen. Letztere können zum Auftauen des Permafrosts und deshalb zu Hanginstabilitäten führen (Williams 1995, Cohen 1997). So ereigneten sich, wahrscheinlich durch eine allmähliche Feuchtigkeitszunahme bedingt, die meisten nacheiszeitlichen Bergstürze und -rutsche in den polnischen Karpaten zwischen 7500 und 6500 v. Chr. (Alexandrowicz \& Alexandrowicz 1999). Eine frühere Phase mit Massenbewegungen fand um 9500 v. Chr. statt, am Übergang vom Spätglazial zum Holozän. Auch in den Alpen ereigneten sich viele Stürze und Rutsche zwischen 9500 und 8000 v. Chr. (Lateltin et al. 1997, Dapples et al. 2003). Der grösste Bergsturz der Alpen, der Flimser Bergsturz (ca. 9 km³ Ablagerungen; Abele 1974), brach wie der Bergsturz von Kandersteg im Boreal um 7900-7000 v. Chr. nieder (von Poschinger \& Haas, 1997). Viele Hinweise deuten darauf hin, dass diese frühholozänen Bergstürze durch starke und teilweise abrupte Anstiege der (Sommer)Temperaturen verursacht wurden.

In letzter Zeit sind erste z.T. quantitative Versuche zur Abschätzung der Auswirkung von Klimaänderungen auf Massenbewegungen in Gebirgen unternommen worden (z.B. Cohen 1997, Buma \& Dehn 1998; Dehn \& Buma 1999). Paläoklimatische, sedimentologische und paläoökologische Daten bieten eine gute Gelegenheit, solche Modelle zu überprüfen. Um Rückschlüsse auf die potentielle Gefährdung von Siedlungsräumen zu ermöglichen, erscheint es in Anbetracht der prognostizierten globalen Klimaänderung eine dringliche Aufgabe, den Zusammenhang zwischen Klimawechsel und Massenbewegungen in den Alpen besser zu verstehen.

\section{Verdankung}

Dank gebührt der BLS Alp Transit AG, welche uns die Einwilligung zur Ausführung der vorliegenden Abklärungen an Bohrkernen und den Zugang zur Baustelle gewährte. Für die Probeentnahme danken wir D. Gutscher (Archäologischer Dienst des Kantons Bern) und für die Herstellung der Figuren 1, 2 und 3 E. Schranz (Archäologischer Dienst des Kantons Bern). Unser Dank geht auch ans Physikalische Institut der Universität Bern, an T. Goslar, Poznan Radiocarbon Laboratory, an M. Suter (Institut für Teilchenphysik, ETH Zürich) sowie an A. Keller (Radiokarbonlabor des Geographischen Instituts der Universität Zürich) für die Radiokarbondatierungen. Ganz herzlich bedanken wir uns bei Chr. Bigler für die (leider erfolglose) Suche nach Kieselalgen und bei F. Oberli für die Aufbereitung der Pollenproben. Die Studie und die Radiokarbondatierungen sind teilweise vom Archäologischen Dienst des Kantons Bern finanziert worden. Wir danken den Rezensenten J.-N. Haas und W. Wildi, dem Redaktor D. Ariztegui und dem Chefredaktor S. Bucher für die wertvollen Ratschläge zur Verbesserung des Manuskripts. 


\section{LITERATURVERZEICHNIS}

Abele, G. 1974: Bergstürze in den Alpen, ihre Vorbereitung, Morphologie und Folgeerscheinungen. Wissenschaftliche Alpenvereinshefte, 25, 1-165.

Alexandrowicz, S. W. \& Alexandrowicz, Z. 1999: Recurrent Holocene landslides: a case study of the Krynica landslide in the Polish Carpathians. Holocene 9, 91-99.

Ammann, B., Gaillard, M.-J. \& Lotter, A. F. 1996: Switzerland. In: Berglund B.E., Birks H.J.B., Ralska-Jasiewiczowa M. \& Wright H.E. (Eds.): Palaeoecological events during the last 15000 years: regional syntheses of palaeoecological studies of lakes and mires in Europe, 647-666, J. Wiley \& Sons, Chichester.

BECK, P. 1929:Vorläufige Mitteilung über die Bergstürze und den Murgang im Kandertal (Berner Oberland). Eclogae geol. Helv. 22/2, 155-159.

BECK, P. 1952: Neue Erkenntnisse über die Bergstürze im Kandertal. Eclogae geol. Helv. 45, 277-280.

Becker, A., Davenport, C. A., Haeberli, W., Burga, C., Perret, R., Flisch, A. \& Keller, W. A. 2000: The Fulnau landslide and former Lake Seewen in the northern Swiss Jura Mountains. Eclogae geol. Helv. 93 , 291-305.

BRADSHAW, R. H. W. \& WEBB, T. I. 1985: Relationships between contemporary pollen and vegetation data from Wisconsin and Michigan, USA. Ecology $66,721-737$.

Buma, J. \& DeHn, M. 1998: A method for predicting the impact of climate change on slope stability. Environ. Geol. 35, 190-196.

CoHen, S. J. 1997: What if and so what in northwest Canada: Could climate change make a difference to the future of the Mackenzie basin? Arctic 50, 293-307.

Dapples, F., Oswald D., Raetzo, H., Lardelli, T., Zwahlen, P.:2003: New records of Holocene landslide activity in the Western and Eastern Swiss Alps: Implication of climate and vegetation changes. Eclogae geol. Helv. 96, 1-9.

DeHn, M. \& Buma, J. 1999: Modelling future landslide activity based on general circulation models. Geomorphology 30, 175-187.

Frey, W. \& Lösch, R. 1998: Lehrbuch der Geobotanik. Gustav Fischer, Stuttgart, 436pp.

Furrer, H., Huber, K., Adrian, H., Baud, W., Flück, W., Preiswerk, C. Schuler, P. \& ZwAhlen, P. 1993: Geologischer Atlas der Schweiz, Blatt 87, Adelboden. Ittigen, Bundesamt für Wasser und Geologie BWG.

Guiot, J., Harrison, S. P. \& Prentice, I. C. 1993: Reconstruction of Holocene Precipitation Patterns in Europe Using Pollen and Lake-Level Data. Quaternary Research 40, 139-149.

HAAS, J.N., 1999. Charophyte population dynamics during the Late Quaternary at Lake Bibersee, Switzerland. Australian Journal of Botany, 47: 315-324.

HaAs, J.N., Richoz, I. TinneR, W. \& Wick, L. 1998: Synchronous Holocene climatic oscillations recorded on the Swiss Plateau and at timberline in the Alps. The Holocene 8, 301-309.

HantKe, R. 1978: Eiszeitalter 1. Die jüngste Erdgeschichte der Schweiz und ihrer Nachbargebiete, 1. Ott Verlag, Thun, 1-468 p.

Heim A. 1932: Bergsturz und Menschenleben. Vierteljahressschr. Natf. Ges. Zürich, 218pp

Hermanns, R. L., Trauth, M. H., Niedermann, S., McWilliams, M. \& Stre CKER, M. R. 2000: Tephrochronologic constraints on temporal distribution of large landslides in northwest Argentina. J. Geol. 108, 35-52.

KöPpen, W. P., 1931. Grundriss der Klimakunde. Walter de Gruyter, Berlin, 388pp.

Kutzbach, J. E. \& WebB III, T. 1993: Conceptual Basis for Understanding Late-Quaternary Climates. In: Wright H.E. JR., KutzBach J.E., WebB T. III, Ruddiman W.F., Street-Perrott F.A. \& Bartlein P.J (Eds.):Global Climates since the Last Glacial Maximum, 5-11, University of Minnesota Press, Minneapolis.

Lateltin, O., Beer, C., Raetzo, H. \& Caron, C. 1997: Landslides in Flysch terranes of Switzerland: Causal factors and climate change. Eclogae geol. Helv. 90, 401-406.

LOTTER, A. F. 1999: Late-glacial and Holocene vegetation history and dynamics as evidenced by pollen and plant macrofossil analyses in annually laminated sediments from Soppensee (Central Switzerland). Vegetation History and Archaeobotany 8, 165-184.

Moore, P. D., WebB, J. A. \& Collinson, M. E. 1991: Pollen analysis. Blackwell Scientific Publications. Second Edition, London, 216pp.

Muscheler, R., Beer, J., Wagner, G. \& Finkel, R. C. 2000: Changes in deep- water formation during the Younger Dryas event inferred from ${ }^{10} \mathrm{Be}$ and ${ }^{14} \mathrm{C}$ records. Nature 408, 567-570.

Reille, M. 1992: Pollen et spores d'Europe et d'Afrique du nord. Laboratoire de Botanique hostorique et Palynologie, Marseille, 520pp.

Schoch, W., PAwlik, B. \& Schweingruber, F. H. 1988: Botanische Makroreste: ein Atlas zur Bestimmung häufig gefundener und ökologisch wichtiger Pflanzensamen. Paul Haupt, Bern, 227pp.

Schwartz, M. W. 1989: Predicting tree frequencies from pollen frequency: an attempt to validate the $\mathrm{R}$ value method. New Phytologist 112, 129-143.

Schweingruber, F. H., 1990: Mikroskopische Holzanatomie. Eidgenössische Forschungsanstalt für Wald, Schnee und Landschaft (WSL), Birmensdorf, 226pp.

SteIgeR, P. 1995: Wälder der Schweiz. Ott Verlag, Thun, 360pp.

STOCKMARR, J. 1971: Tablets with spores used in absolute pollen analysis. Pollen et Spores 13, 615-621.

Stuiver, M., Reimer, P. J., Bard, E., Beck, J. W., Burr, G. S., Hughen, K. A., Kromer, B., McCormac, G., van der Plicht, J. \& Spurk, M. 1998 INTCAL98 radiocarbon age calibration, 24,000-0 cal BP. Radiocarbon 40, 1041-1083.

SugitA, S. 1994: Pollen representation of vegetation in Quaternary sediments: theory and method in patchy vegetation. Journal of Ecology 82, 881-897.

Sugita, S., MacDonald, G. M. \& Larsen, C. P. S. 1997: Reconstruction of fire disturbance and forest succession from fossil pollen in lake sediments: potential and limitations. In: Clark J.S., CACHIER H., Goldammer J.G. \& STOCKS B.J. (Eds.): Sediment Records of Biomass burning and Global Change, 387-412, Springer, Berlin.

Tibaldi, A., Ferrari, L. \& Pasquare, G. 1995: Landslides Triggered by Earthquakes and Their Relations with Faults and Mountain Slope Geometry - an Example from Ecuador. Geomorphology 11, 215-226.

Tinner, W., Conedera, M., Ammann, B., Gäggeler, H. W., Gedye, S., Jones, R. \& SÄgesser, B. 1998: Pollen and charcoal in lake sediments compared with historically documented forest fires in southern Switzerland since AD 1920. The Holocene 8, 31-42.

Tinner, W. \& LotTer, A. F. 2001: Central European vegetation response to abrupt climate change at $8.2 \mathrm{ka}$. Geology 29, 551-554.

TinneR, W \& Theurillat J. P. 2003: Uppermost limit, extent, and fluctuations of the timberline and treeline ecocline in the Swiss Central Alps during the past 11,500 years. Arct. Antarct. Alp. Res. 35: 158-169.

Tinner, W., Hubschmid, P., Wehrli, M., Ammann, B. \& Conedera, M. 1999: Long-term forest fire ecology and dynamics in southern Switzerland. Journal of Ecology 87, 273-289.

Tinner, W., Conedera, M., Gobet, E., Hubschmid, P., Wehrli, M. \& AmMANN, B. 2000: A palaeoecological attempt to classify fire sensitivity of trees in the southern Alps. The Holocene 10, 565-574.

Tinner, W. \& Ammann, B. 2001: Timberline paleoecology in the Alps. PAGES News 9, 9-11

Tinner, W., Conedera, M., Ammann, B. \& Lotter, A. F.: Fire ecology north and south of the Alps since the last ice-age. The Holocene, in review.

Trauth, M. H., Alonso, R. A., Haselton, K. R., Hermanns, R. L. \& StreCKER, M. R. 2000: Climate change and mass movements in the NW Argentine Andes. Earth and planet. Sci. Lett. 179, 243-256.

VAN DER KNAAP, W. O. \& AMmANn, B. 1997: Depth-age relationships of 25 well-dated Swiss Holocene pollen sequences archived in the Alpine palynological data-base. Revue paléobiologie Genève 16, 433-480.

von Poschinger, A. \& HAAs, U. 1997: Der Flimser Bergsturz, doch ein warmzeitliches Ereignis? Bull. angew. Geol. 2/1, 35-46.

Welten, M. 1982: Vegetationsgeschichtliche Untersuchungen in den westlichen Schweizer Alpen: Bern-Wallis. Denkschriften Schweizerische Naturforschende Gesellschaft 95, 1-104.

WiCK, L. \& TinNER, W. 1997: Vegetation changes and timberline fluctuations in the Central Alps as indicator of Holocene climatic oscillations. Arctic and Alpine Research 29, 445-458.

Williams, P. J. 1995: Permafrost and Climate-Change - Geotechnical Implications. Philos. Trans. R. Soc. Lond. Ser. A-Math. Phys. Eng. Sci. 352 347-358.

ZOLLER, H. \& HAAs, J. N. 1995: War Mitteleuropa ursprünglich eine halboffene Weidelandschaft oder von geschlossenen Wäldern bedeckt? Schweiz. Z. Forstwes. 146, 321-354

Manuskript eingegangen 9. Februar 2004

Revidierte Fassung angenommen 17. Januar 2005 


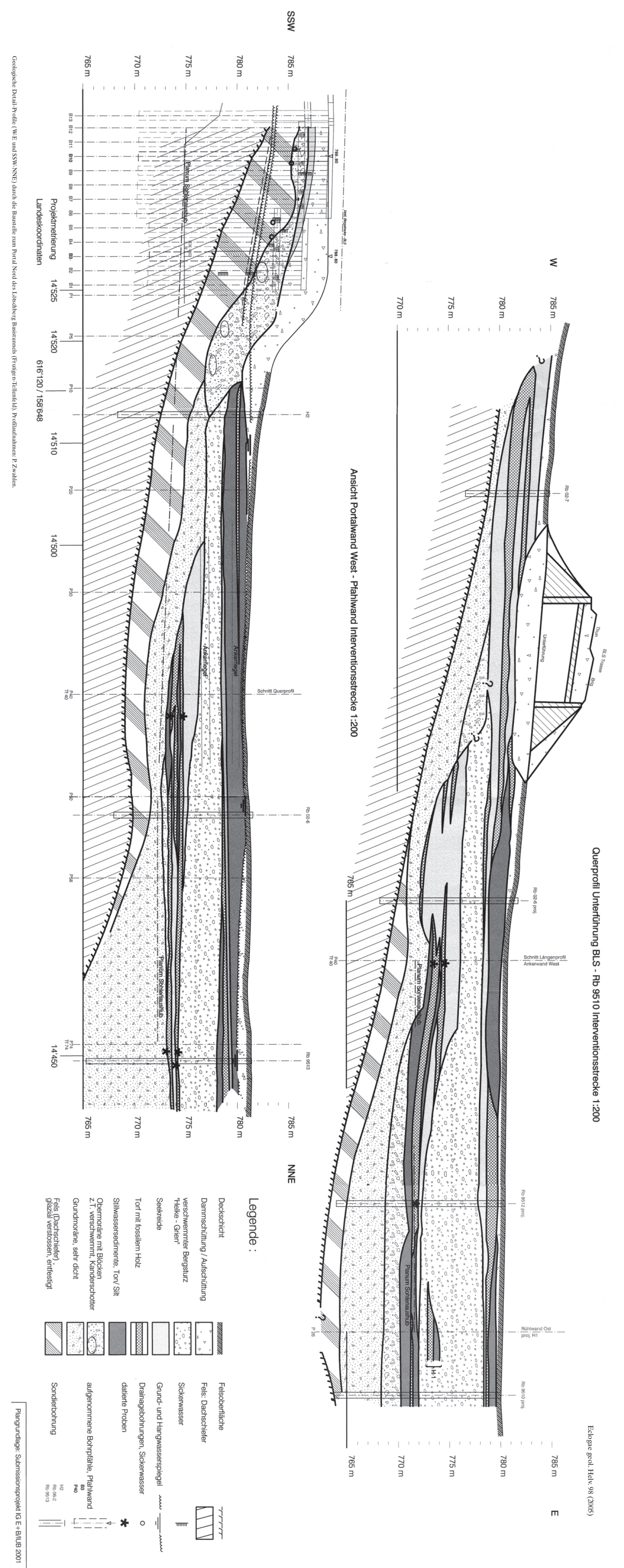

